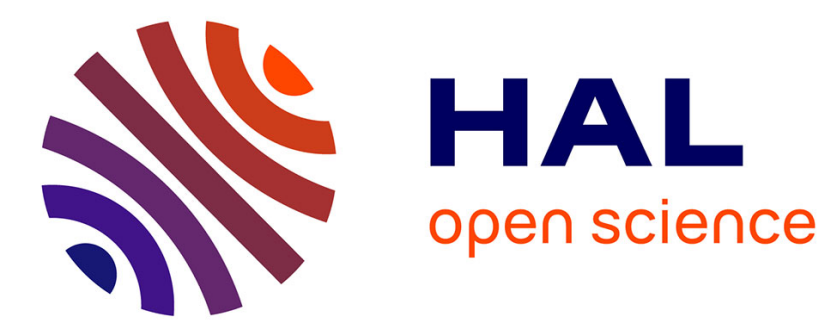

\title{
Sur la deuxième approximation de la méthode de Born
} Jacques Winter

\section{To cite this version:}

Jacques Winter. Sur la deuxième approximation de la méthode de Born. J. Phys. Radium, 1935, 6 (2), pp.71-72. 10.1051/jphysrad:019350060207100 . jpa-00233300

\section{HAL Id: jpa-00233300 https://hal.science/jpa-00233300}

Submitted on 1 Jan 1935

HAL is a multi-disciplinary open access archive for the deposit and dissemination of scientific research documents, whether they are published or not. The documents may come from teaching and research institutions in France or abroad, or from public or private research centers.
L'archive ouverte pluridisciplinaire HAL, est destinée au dépôt et à la diffusion de documents scientifiques de niveau recherche, publiés ou non, émanant des établissements d'enseignement et de recherche français ou étrangers, des laboratoires publics ou privés. 


\title{
SUR LA DEUXIĖME APPROXIMATION DE LA MÉTHODE DE BORN
}

\author{
Par JacQues WINTER.
}

\begin{abstract}
Sommaire. - On discute la validité de la deuxième approximation de la méthode de Born, dans le problème du choc élastique d'électrons rapides sur les atomes neutres. On aboutit à la conclusion que cette approximation est très douteuse, et ne peut sans doute pas servir à corriger l'erreur réellement commise.
\end{abstract}

But de cette note. - On appelle méthode de Born, en physique quantique, une application de la méthode des approximations successives dans un cas où le critère de convergence fait défaut : celui de la diffusion d'une onde plane par un champ atomique, lorsque la quantité de mouvement $k$ de l'onde augmente indéfiniment. On sait que sil'atome se réduit à son noyau, la première approximation est valable, alors que la deuxième est infinie et n'a plus de sens. Nous avons, dans notre thèse, (') démontré la validité de la première approximation, sans mettre en cause la deuxième, tout au moins dans la mesure où elle se ramène à la loi de Rutterford qui donne des flux diffusés en $1 / \sin ^{*}\left(\begin{array}{l}0 \\ \overline{2}\end{array}\right),(\theta$ étant l'angle de la direction où on observe la diffusion avec la direction de propagation de l'onde). Notre démonstration s'applique lorsque le rayon de l'atome $r_{0}$ devient infini (2), cas où, nous venons de le dire, la méthode de Born devient très sujette à caution. Mais lorsque $r_{0}$ est de l'ordre de $a\left(a=\frac{h^{2}}{4 \pi^{2} m e^{2}}\right)$, la deuxième approximation de Born peut devenir effectivement beaucoup plus petite que la première.

Avons-nous le droit d'en conclure qu'elle est valable, c'est-à-dire, qu'elle représente bien la partie principale de l'erreur commise en s'arrêtant à la première approximation? Nous avons laissé cette question sans réponse nette et nous voulons la discuter ici.

Le critère de convergence. - On sait que la $n^{\text {iène }}$ approximation s'obtient en fonction de la $(n-1)$ ième par la formule de récurrence

$$
\begin{gathered}
\Psi_{n}(x, y, z)=-\frac{1}{4 \pi} \cdot \frac{2 Z}{a} \int_{r^{\prime}<r_{0}}\left(\frac{1}{r^{\prime}}-\frac{1}{r_{0}}\right) \frac{\mathrm{e}^{-\mathrm{i} k\left|r-r^{\prime}\right|}}{\left|r-r^{\prime}\right|} \\
\Psi_{n-1}\left(x^{\prime}, y^{\prime}, z^{\prime}\right) \mathrm{d} x^{\prime} \mathrm{d} y^{\prime} \mathrm{d} z^{\prime} .
\end{gathered}
$$

(1) Paris, Masson, 1934, Ann. de Phys., 1934, t. 2, p. 4:53.

(2) On représente le champ de l'atome par la formule :

$$
V=\frac{Z e}{r}\left(1-\frac{r}{r_{0}}\right)
$$

pour $r<r_{0}$, et 0 pour $r>r_{0}$ ( $V$ est le potentiel $)$. On n'étudie que la diffusion élastique. $Z$ nombre atomique, $e$ charge de l'électron, $r$ coordonnée polaire usuelle, $h$ constante de Planck. $m$ masse de l'électron.
En nous inspirant du raisonnement de M. Picard (') nous voyons qu'il y aura convergence, comme une progression géométrique de raison $\lambda \gamma, \gamma$ étant une borne supérieure de $V$ et $\lambda$ étant la borne supérieure dans la sphère $r<r_{0}$, de l'intégrale.

$$
\frac{1}{2 \pi a e} \iiint_{r^{\prime}<r_{0}} \uparrow\left(x, y, z, x^{\prime}, y^{\prime}, z^{\prime}\right) \mathrm{d} x^{\prime} \mathrm{d} y^{\prime} \mathrm{d} z^{\prime}
$$

$\varphi$ étant une fonction positive, toujours égale ou supérieure au module de $\frac{\mathrm{e}^{-\mathrm{i} k\left|r-r^{\prime}\right|}}{\left|r-r^{\prime}\right|}$. La valeur la plus favorable de $\varphi$ sera $\frac{1}{\left|r-r^{\prime}\right|}$.

Une première difficulté consiste en ce que $V$ n'est pas bornée supérieurement. Mais nous insisterons surtout sur ceci : $n i \lambda, n i$ y ne dépendent de $k$ (en admettant $\left({ }^{2}\right)$ qu'on puisse définir $\gamma$, en éliminant la partie centrale du champ, c'est-à-dire le voisinage immédiat de l'origine) et le raisomment de $\mathbf{M}$. Picard ne pourrait subsister que si $Z$ était assez petit pour que $\lambda \gamma<1$, ce qui ne sera jamais réalisé (3). La présence de $\mathbf{e}^{-i k\left|r-r^{\prime}\right|}$ ne nous permet pas de conclure que $\Psi_{n}$ sera de l'ordre de grandeur de $\left(\frac{Z}{2 \pi a k}\right)^{n}$, car $\Psi_{n-1}$ peut contenir une autre exponentielle qui compensera l'influence de la première sur une partie du domaine d'intégration. Le rapport des modules de $\Psi_{n}$ et $\Psi_{n-1}$ pourra dépendre de $k$ d'une manière plus compliquée, qui dépendra du domaine d'intégration $r_{0}$, et de l'ordre d'approximation $n$. En prenant avec M. Distel $\left(^{4}\right)$ les coordonnées $k x$, $k y, k z$, on fait bien appparaître $\frac{1}{k}$ dans la fonction $V$, mais le domaine d'intégration devient $r^{\prime}<k r_{0}$, de sorte que la difficulté s'introduit d'une autre manière : par l'extension indéfinie du domaine d'intégration.

(1) Leçons sur quelques problèmes aux limites de la théorie des équations différentielles, Paris, 1928, p. 127 et suiv.

(2) Ce qui nécessiterait, dailleurs, une discussion.

(3) En prenant $r_{0}=a$, et pour $\gamma$ la valeur très favorable $\frac{Z e}{a}$ on a, $\lambda \gamma=Z$.

(4) Z. physik, 1932, t. 74, p. 785 . 
La méthode rigoureuse. - Nous ne reprendrons pas (1) l'exposé de la méthode rigoureuse, qui consiste à développer l'onde plane en fonctions sphériques, et a étudier l'action du champ atomique sur chaque élément de décomposition. Nous avons montré qu'en prenant $\frac{1}{k r_{0}}$ et $\frac{Z}{a k}$ pour infiniment petits principaux, le flux diffusé avait bien l'expression donnée par Rutherford, avec une correction relative de l'ordre de $\frac{1}{\sqrt{k r_{0}}}$ (si on faisait croître indéfiniment $r_{0}$, cette correction disparaîtrait, mais il en subsisterait d'autres, de l'ordre de $\left.\frac{Z}{a k}\right)$. Cette correction provient detous les éléments de décomposition de l'onde plane, qui entrent en ligne de compte, et ceux-ci sont au nombre de $k r_{0}$. La complexité du problème tient au fait que, $k$ augmentant, il faut prendre un nombre de plus en plus grand d'éléments, dans la décomposition de l'onde plane. C'est là, le fait essentiel: l'expression analytique de la fonction à étudier se modifie lorsque $k$ augmente, alors que la méthode des approximations successives part toujours de la mème fonction, lorsqu'on veut voir comment diminue l'erreur, en faisant tendre vers zéro le paramètre arbitraire qui multiplie le terme perturbateur. Si la fonction de départ (l'approximation zéro), varie elle aussi avec le paramètre (c'est le cas ici, puisqu'on part de $\mathbf{e}^{-\mathrm{i} k x}$ et que le paramètre qui tend vers zéro est $\left.\frac{Z}{k a}\right)$, les choses cessent d'ètre simples $\left({ }^{2}\right)$.

Or, la méthode de Born, appliquée au champ $V$, donne pour $\Psi_{1}$ en un point $r, \theta$, très éloigné de l'atome diffuseur :

$$
\Psi_{1}=-\frac{4}{a} \cdot Z \frac{\mathrm{e}^{\mathrm{i} k r}}{r}\left\{\frac{1}{\Delta^{2}}-\frac{\sin \Delta r_{0}}{\Delta^{3} r_{0}}\right\}
$$

avec

$$
\Delta=2 k \sin \frac{\theta}{2}
$$

Le premier terme donne la loi de Rutherford. Le deuxième est (pour $\theta \neq 0$ ) une correction relative de l'ordre de $\frac{1}{k r_{0}}$, et doit certainement ètre négligé, si on ne tient pas compte de la deuxième approximation, qui représente une correction de l'ordre de $\frac{Z}{a k}$. Mais nous

(1) Voir L. de Broglı. Annales de l'instıtut Poincaré, t. 3, 1933, p. 402 et suivantes.

(2) On peut ausi dire nous l'avons vu, que l'intensité de la perturbation tend vers zéro, tandis que son domaine s'accroit indéfiniment. Ce sont des manières équivalentes d'exprimer la difficulté. voyons que l'une et l'autre de ces deux corrections ne peuvent donner le terme en $\frac{1}{\sqrt{k r_{0}}}$, obtenu en première approximation.

Conclusion. $-1^{\circ}$ La formule de Born (en première approximation) et la formule de Rutherford ne diffèrent que par un terme qui doit être certainement négligé. Nous avons done eu tort d'espérer (1) que la formule de Born puisse avoir un domaine de validité plus grand que celui de la formule de Rutherford: 2。 Passons maintenant à la deuxième approximation de la méthode de Born. Lorsque $r_{0}$ était infini, elle élait trop grande pour ètre utilisable et pour avoir un sens. Si $r_{0}$ estéquivalentà $a$ nous voyons qu'elle devient trop petile. Ceci serait évident si on pouvait admettre qu'elle est de l'ordre de $\left(\frac{Z}{a k}\right)^{2}$. Mais nous avons vu qu'une telle affirmation n'est pas fondée. Mais elle contient certainement $Z^{2}$ en facteur, alors que la correction en $\frac{1}{\sqrt{k r_{0}}}$ que nous avons obtenue ne contient que $Z$ en facteur. Ainsi elles ne peuvent certainement pas se compenser.

Il ne resterait donc qu'une chance de validité pour la deuxième approximation de Born : nous n'avons donné qu'une limite supérieure de l'erreur en première approximation. Il se pourrait qu'une compensation ait lieu entre les différentes quantités qui la composent. La correction réelle deviendrait ainsi d'un ordre de grandeur inférieur et notre objection tomberait. Mais rien ne nous permet d'espérer une telle compensation. Elle pourrait évidemment avoir lieu pour une forme particulière de la fonction $V$. Mais si on modifiait $V$, dans la partie périphérique de l'atome, elle disparaîtrait $\left({ }^{2}\right)$. Or le potentiel $V$, dans la périphérie de l'atome, est complètement inconnu. La deuxième approximation de la méthode de Born nous paraît donc tout à fait-sujette à caution, même lorsque $\frac{Z}{a k} \leqslant 1$ et $r_{0} \sim a$.

(1) Nous avons dit (Thèse, p. 106) que les conditions de validité de la formule de Rutherford devenaient trop restritives si on les appliquait à la formule de Born. A première vue, c'était possible. Mais nous n'avions pas remarqué qu'elles ne différaient que par un terme négligeable.

${ }^{2}{ }^{2}$ On peut s'en rendre compte en examinant la figure (19), p. $10^{:}$, de notre thèse. La correction est constituée par une série de polynômes, de Legendre $P_{n}$, dont les coefficients sont, aux poin $\left(1^{\circ}\right)$ et $\left(3^{\circ}\right)$, la courbe $\left(3^{\circ}\right)$ devant ètre prolongée par l'axe des abscisses. Si on modifie $\Gamma^{\circ}$, on déformera la courbe $\left(3^{\circ}\right)$, sans que l'allure de sa dérivée au voisinage de l'axe $0 \delta$ en soit changée. La modification due à cette déformation sera encore de l'ordre de $\frac{1}{\sqrt{k r_{u}}}$, mais ne sera pas nulle. 